\title{
Hepatopulmonary Syndrome: Monitoring at Your Fingertip
}

\author{
Michael J. Krowka
}

Published online: 30 March 2011

(C) Springer Science+Business Media, LLC 2011

Hepatopulmonary syndrome (HPS) is the triad of arterial hypoxemia due to pulmonary vascular dilatation induced by liver disease [1]. Diagnosis of HPS relies on measurement of arterial blood gas (ABG) to determine the severity of hypoxemia, whereas vascular dilatation is demonstrated by positive contrast-enhanced transthoracic echocardiography $(\mathrm{CE})$.

An invasive test, $\mathrm{ABG}$ is usually obtained via a radial artery stick. Approximately $5 \mathrm{cc}$ arterial blood is drawn into a sealed, heparinized syringe and inserted into a blood-gas analyzer within $30 \mathrm{~min}$ of collection. Oxygenation is quantified by two measures. $\mathrm{PaO}_{2}$ in $\mathrm{mmHg}$ (partial pressure of oxygen dissolved in the plasma; normal considered by most authorities $>80 \mathrm{mmHg}$ at sea level) or the calculated alveolar-arterial oxygen gradient $\left(\mathrm{A}-\mathrm{a} \mathrm{O}_{2}\right.$ grad) in $\mathrm{mmHg}$ (difference between alveolar oxygen and $\mathrm{PaO}_{2}$; normal is age-dependent and $<15-20 \mathrm{mmHg}$ ). $\mathrm{A}-\mathrm{a} \mathrm{O}_{2}$ grad is the more sensitive measure that takes into account the degree of ventilation within the lung [1], but it is not calculated in all pulmonary function laboratories. Importantly, oxygenation in patients with HPS can differ significantly (up to $20 \mathrm{~mm}$ $\mathrm{Hg}$ ) as body position changes (orthodeoxia) with $\mathrm{PaO}_{2}$ supine $>$ sitting $>$ standing and is worse with exercise [1].

Fortunately, an easier measure of arterial oxygenation has evolved-pulse oximetry [2]. Simply, pulse oximetry allows noninvasive measurement of arterial hemoglobin saturation by oxygen, without the risks associated with arterial puncture [2]. Via a sensor placed on the finger tip (earlobe or forehead as needed), pulse oximetry determines the percentage of oxygen that saturates circulating

M. J. Krowka ( $₫)$

Division of Pulmonary and Critical Care Medicine, Mayo Clinic, Rochester, MN 55905, USA

e-mail: Krowka@mayo.edu hemoglobin $\left(\mathrm{SaO}_{2}\right)$ [2]. This measurement is based upon light absorption within arterial blood at specific wavelengths; red (640 nm) and infrared (940 nm) [3]. Unfortunately, this method cannot distinguish between oxygen and carbon monoxide attached to hemoglobin [2]. The measurement can be one-time or continuous over time. The latter is particularly important during sleep in the setting of advanced liver disease, because $\mathrm{SaO}_{2}$ can frequently drop below $90 \%$ [4].

Is there a close relationship between $\mathrm{PaO}_{2}$ and $\mathrm{SaO}_{2}$ ? Indeed there is via the oxyhemoglobin dissociation curve [2]. Although beyond the scope of this editorial, recall this relationship is sigmoid, not linear. Therefore, under normal conditions, big change in $\mathrm{PaO}_{2}(100 \rightarrow 50 \mathrm{mmHg})$ can occur in association with a relatively small change in $\mathrm{SaO}_{2}$ $(98 \rightarrow 89 \%)$.

By any measure $\left(\mathrm{PaO}_{2}\right.$ or $\mathrm{A}-\mathrm{a} \mathrm{O}_{2}$ grad $)$ hypoxemia has common causes (40-50\%) in advanced liver disease; COPD, hepatic hydrothorax, interstitial lung disorders, massive ascites, or obesity, to name a few. Hypoxemia due to HPS is of particular interest because oxygenation can worsen after the diagnosis is made, but it is unclear how rapidly and why change occurs without obvious deterioration in hepatic function. Such change is not trivial, because current organ procurement/liver allocation policy states that once $\mathrm{PaO}_{2}$ is less than $60 \mathrm{mmHg}$ (sitting position, breathing room air) MELD (model for end-stage live disease) exception may be granted to enhance priority for pediatric and adult liver transplantation [5]. How can change in oxygenation be sequentially monitored in HPS patients?

Kochar and colleagues in this issue [6] describe pulse oximetry to assess change in oxygenation over time in HPS. They reported periodic, one-time $\mathrm{SaO}_{2}$ measurements obtained in the sitting position, breathing room air. Retrospectively, worsening oxygenation was documented 
periodically over a 36 month period. Unfortunately, they probably excluded HPS patients who had concomitant pleural effusions ( $>20 \%$ pleural space), reduced ejection fraction, and patients with more than mild expiratory airflow obstruction $\left(\mathrm{FEV}_{1} / \mathrm{FVC}<70 \%\right)$. Up to $30 \%$ of HPS patients can have other pulmonary issues, further challenging their liver transplant management [1].

A few observations about their data merit comment. Baseline ABG data demonstrated that most patients had mild HPS with 13 of 22 patients having $\mathrm{PaO}_{2}>80 \mathrm{mmHg}$ (a level many clinicians might interpret as normal). Only two of their patients had baseline $\mathrm{PaO}_{2}<60 \mathrm{mmHg}$ and their particular $\mathrm{PaO}_{2} / \mathrm{SaO}_{2}$ values were $53 / 89 \%$ and $52 / 90 \%$, respectively. The lowest $\mathrm{SaO}_{2}$ in those with $\mathrm{PaO}_{2}>80 \mathrm{mmHg}$ was $95 \%$. If $\mathrm{SaO}_{2}<95 \%$ is observed in a liver transplant candidate with an established HPS diagnosis, periodic ABG should be obtained every 6 months to monitor further change, in my opinion.

Using pulse oximetry as an inexpensive screen for HPS is another issue. It would seem reasonable that when spot check $\mathrm{SaO}_{2}$ is less than $95 \%$ one should have increasing concern about HPS as a cause of hypoxemia. Certainly in that case contrast-enhanced echocardiography and ABG should be done.

At my institution $\mathrm{CE}$ is routinely done in the liver transplant evaluation to screen for pulmonary vascular dilatations. Positive $\mathrm{CE}$ and normal oxygenation by $\mathrm{ABG}$ is not uncommon $(\sim 40-50 \%)$. Kochar's data are similar and suggest no change in $\mathrm{SaO}_{2}$ over time in such individuals (up to three years). Intuitively, patients do not transition from positive $\mathrm{CE} /$ normal oxygenation to positive $\mathrm{CE}$ and severe hypoxemia in a "quantum jump". Continued monitoring with pulse oximetry in such patients would be prudent. We do not know which of those patients will truly evolve into the clinically significant syndrome, but I would guess about $10 \%$.

Pulse oximetry caveats? Keep in mind two common situations that may alter accurate oximetry measurements-deep breathing $\left(\mathrm{SaO}_{2}\right.$ due to hyperventilation may increase up to $4 \%$ ) and smoking (carbon monoxide attached to hemoglobin is included in the total $\mathrm{SaO}_{2}$ percent)—so the true oxygenation abnormality is underestimated in such patients by several percent [2]. Another condition, methemoglobinemia caused by several drugssulfonamides and topical benzocaine to name a couple common ones-will adversely affect $\mathrm{SaO}_{2}$ (a very low reading) because oxidized iron in the hemoglobin cannot bind oxygen [2]. An ABG clarifies the suspected carboxy and methemoglobin issues. Digital clubbing does not affect $\mathrm{SaO}_{2}$; nail polish can. Standard deviation of measurements is approximately $1 \%$ (Table 1 ).

Being mindful of the cost of medical care (an ABGarterial puncture plus analysis—costs approximately \$300),
Table 1 Monitoring hepatopulmonary syndrome

Diagnosis confirmed by arterial blood gas
Invasively measures partial pressure of oxygen $\left(\mathrm{PaO}_{2}\right)$
Finger pulse oximetry
Noninvasively measures hemoglobin saturation by oxygen $\left(\mathrm{SaO}_{2}\right)$
$\mathrm{SaO}_{2}$ overestimates oxygenation in smokers because of carbon
monoxide
$\mathrm{Small}$ change in $\mathrm{SaO}_{2}$ associated with large change in $\mathrm{PaO}_{2}$
$\mathrm{SaO}_{2}<95 \%$ merits $\mathrm{ABG}$, especially if MELD exception to be
considered

the use of pulse oximetry makes sense. Perhaps the expense and diagnostic use of contrast-enhancement during transthoracic echocardiography (which requires intravenous access) can be reserved for those with concerning oximetry results $\left(\mathrm{SaO}_{2}<95 \%\right)$.

A final HPS question beyond pulse oximetry should be pondered on the basis of the data in this paper. In view of the frequent positive $\mathrm{CE}$ findings in patients with advanced liver disease, should the definitive oxygenation criteria for HPS be modified to reflect what is truly a clinical or symptomatic syndrome?, Consider patient \#4 in the Kochar study with a positive $\mathrm{CE}$ (we do not know to what degree), $\mathrm{PaO}_{2}$ of $95 \mathrm{mmHg}$ and $\mathrm{A}-\mathrm{a}$ grad of $16.5 \mathrm{mmHg}$. Indeed, the patient has HPS by current criteria. Might this be better-termed "subclinical" or forme fruste HPS? Perhaps it is time to revisit the HPS definition.

In summary, oxygenation determined via $\mathrm{ABG}$ in the sitting position, breathing room air, remains the definitive diagnostic criteria for HPS and provides the key MELD exception data point $\left(\mathrm{PaO}_{2}<60 \mathrm{~mm} \mathrm{Hg}\right)$. Monitoring $\mathrm{SaO}_{2}$ via fingertip pulse oximetry is quick, with no expense, noninvasive, accurate for the most part, quite reproducible, and seems useful to estimate arterial oxygenation trend. However, an ABG remains the confirmatory study to determine the specifics and severity of arterial hypoxemia associated with liver disorders.

\section{References}

1. Rodriguez-Roisin R, Krowka MJ. Current concepts: hepatopulmonary syndrome-a liver-induced lung vascular syndrome. $N$ Engl J Med. 2008;358:2378-2387.

2. Teff TA. Pulse oximetry: the fifth vital sign. Chest. 1988;94:227.

3. Jubran A. Pulse oximetry. Intensive Care Med. 2004;30:2107.

4. Okcay A, Somers V, Wiesner RH, Krowka MJ. Prevalence and clinical predictors of abnormal overnight oximetry in liver transplant candidates. Hepatology. 2009;50:588. (abstract).

5. United Network for Organ Sharing (UNOS). Liver Allocation Policy 3.6.1. http://www.optn.transplant.hrsa.gov/policies. November 9, 2010.

6. Kochar R, Tanikella R, Fallon MB. Serial pulse oximetry in hepatopulmonary syndrome. Dig Dis Sci. 2011; (this issue). doi: 10.1007/s10620-011-1600-7. 